\title{
Childhood Cancer Incidence and Survival 1985-2009, Khon Kaen, Thailand
}

\author{
Surapon Wiangnon ${ }^{1 *}$, Arunee Jetsrisuparb ${ }^{1}$, Patcharee Komvilaisak ${ }^{1}$, Krittika \\ Suwanrungruang ${ }^{2}$
}

\begin{abstract}
Background: The Khon Kaen Cancer Registry (KKCR) was established in 1984. Previous population-based incidences and survivals of childhood cancer in Thailand were determined using a short cancer registration period. Materials and Methods: Data were retrieved of all children residing in Khon Kaen, between 0-15 years, diagnosed as having cancer and registered in the KKCR (1985-2009). The follow-up censored date was December 31, 2012. The childhood cancers were classified into 12 diagnostic groups, according to the International Classification of Childhood Cancer. The incidence was calculated by the standard method. Survival of childhood cancer was investigated using the KKCR population-based registration data and overall survival calculated using the Kaplan Meier method. Results: In the study period, 912 newly diagnosed cases of childhood cancer were registered. The respective mean and median age was $6.4(\mathrm{SD}=4.6)$ and $6(0-14)$ years. The age-peak for incidence was $0-4$ years. The age-standardized rate (ASR) was 83 per million. Leukemia was the most common cancer $(\mathrm{N}=360$, ASR 33.8) followed by neoplasms of the central nervous system (CNS, $N=150$, ASR 12.8) and lymphoma ( $N=79$, ASR 7.0). The follow-up duration totaled 101,250 months. The death rate was 1.11 per 100 person-months (95\% CI: 1.02 -1.20). The 5-year overall survival was 52\% (95\% CI: 53-56.9) for all cancers. The respective 5-year overall survival for (1) acute lymphoblastic leukemia (ALL), (2) acute non-lymphoblastic leukemia (ANLL), (3) lymphoma, (4) germ cell tumors, (5) renal tumors, (6) retinoblastoma, (7) soft tissue tumors, (8) CNS tumors, (9) bone tumors, (10) liver tumors, and (11) neuroblastoma was (1) $51 \%$, (2) $37 \%$, (3) 63\%, (4) $74 \%$, (5) $67 \%$, (6) $55 \%$, (7) $46 \%$, (8) $44 \%$, (9) $36 \%$, (10) $34 \%$, and (11) $25 \%$. Conclusions: The incidence of childhood cancer is lower than those of western countries. Respective overall survival for ALL, lymphoma, renal tumors, liver tumors, retinoblastoma, soft tissue tumors is lower than that reported in developed countries while survival for CNS tumors, neuroblastoma and germ cell tumors is comparable.
\end{abstract}

Keywords: Incidence - survival - childhood cancer - khon kaen - Thailand

Asian Pac J Cancer Prev, 15 (18), 7989-7993

\section{Introduction}

In Thailand, $21 \%$ of the population is under 15 (National Statistics Office, 2003) and although cancers among this age group are rare, 1,000 were diagnosed with cancer based on the expected incidence (Vattanasapt, 1999; Wiangnon et al., 2003; Thai Pediatric Oncology Group, 2011). Between 2007-8, the respective cancer related mortality rates in Thai children 6-12 and 13-18 was 2.16 and 2.13 per 100,000 (Sutra et al., 2009).

The previous population-based incidences of childhood cancer in Thailand were calculated by extrapolating the incidences from the data, which was limited because of the small number of cancer registries. Recently, the Thai Pediatric Oncology Group (ThaiPOG) reported on the country incidence between 2003 and 2005; but survival was thus limited to children registered during this period.
In addition, the previously reported survival of childhood cancer patients was described with respect to specialized hospitals and/or institutions. The aim of the current investigation was to perform a long-term population-based retrospective study of the incidence of childhood cancer in a homogeneous population in Khon Kaen province.

Registration area and registry procedures: Khon Kaen province is located in northeastern Thailand. The province covers an area of $10,886 \mathrm{~km}^{2}$ with a population of 1.7 million (Figure 1). The Khon Kaen Cancer Registry (KKCR) was established in 1984 at the Faculty of Medicine, Khon Kaen University. The KKCR has been conducting both hospital and population-based registration.

The data on cancer patients was collected using both active and passive methods from all hospitals and private clinics in the province. The registrars regularly 


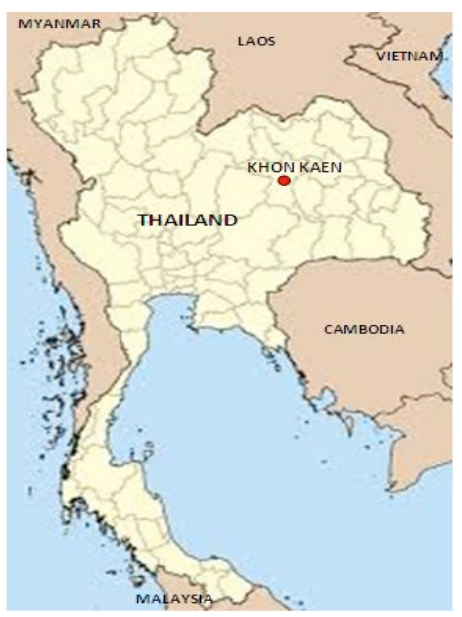

Figure 1. Khon Kaen Map

visit all registered sites. The medical charts of patients diagnosed with cancer were reviewed and the cancer data registered by registry personnel. Any questionable cases were traced to the original resource of information for clarification. Multiple primary cases were checked by physicians using their original records and with any other physicians concerned. Death certificates with a mention of cancer were collected from the population statistics of the office of the Ministry of Interior. The vital status of registered cases has been regularly updated by checking with the population statistics of the office of the Ministry of Interior. To prevent duplication, all data were reviewed and checked with existing registry files before data entry. All the data were verified, checked for duplication coded and entered into the CanReg4 software.

\section{Materials and Methods}

Data on all childhood cancers were retrieved from the KKCR data set for the period 1985-2009. Cancer sites, morphology and behavior were coded according to the International Classification of Diseases for Oncology3rd edition (ICD-O-3) (WHO, 2000). Tumor types were grouped according to the International Childhood Cancer Classification (ICCC) (Kramarova et al., 1996). Both histologically-verified and non-verified patients were included. All patients were observed until the end of December 2012. The aged-standardization rate (ASR) was performed using a direct method with groups $0-14$ years (0-4, 5-9 and 10-14 years) (Parkin et al., 1998). ASRs were calculated by the direct method, using the world standard population and expressed per million persons. ASRs for five-year age groupings (viz., 19851989, 1990-1994, 1995-1999, 2000-2004,2005-2009) were calculated for both boys and girls (Jensen et al., 1991). Five-year cumulative survival rates were calculated using the Kaplan-Meier method. The Generalized Linear Model (GLM) method was applied for analysis based on incidence rates, corresponding p-values.

\section{Population denominators}

The population denominators used for calculation of the incidence rate were (a) the numbers of children from the population censuses in 1990 and 2000 and (b) the estimated projected numbers based on data of 2010 (National Statistic Office, 2003). This provided an annual estimate by age group and sex. Notably, the child population has been continuously decreasing since1980s. According to the 2000 census, 435,369 children were under 15 (25.1\% of the Khon Kaen population) with similar numbers of boys and girls. On average, $30.8 \%$ were under 5,34.8\% were under 10 , and $34.4 \%$ were under 15 (National Statistic Office, 2003) (Table 1).

\section{Results}

There were 912 cases with childhood cancer diagnosed between 1985-2009 (boys $=526$, girls $=386$, ratio 1.4:1). The respective mean and median age was $6.4(\mathrm{SD}=4.6)$ and $6(0-14)$ years. The age-peak for incidence was between 0 and 4 years. The follow-up duration totaled 101,250 months. The death rate was 1.11 per 100 personmonths (95\%CI: 1.02-1.2). The 5-year overall survival was 52\% (95\% CI: 53-56.9) for all cancers (Table 2).

Most of the pediatric cancers were histologicallyconfirmed (83.7\%) but the proportion varied between $52.4 \%$ for cancer of the central nervous system (ICCC group III) and $99.7 \%$ for leukemia (ICCC group I).

The age-standardized rate (1985-2009) was 83 per million. The incidence varied between 58.1 and 99.3 per million according to the 5-year interval. The time trend analysis revealed that childhood cancers increased in boys and girls ( $9 \%, \mathrm{p}=0.004$ and $7 \% \mathrm{p}=0.05$ ) (Table 3 , Figure 2). Leukemia was the most common cancer $(\mathrm{N}=360$, ASR 33.8) followed by neoplasms of the central nervous system (CNS) (N=150,ASR 12.8) and lymphoma $(\mathrm{N}=79$, ASR 7.0). (Table 3) Among the leukemias, acute lymphoblastic leukemia (ALL) was more common than acute non-lymphoblastic leukemia (ANLL) (ratio, 3.9:1). ALL was more common among boys than girls (ASRs 31.1 vs 20.3) while the incidence of ANLL between sexes was not different in both sexes. Non-Hodgkin lymphoma (NHL) is more common than Hodgkin (HD) and Burkitt's lymphoma (ASRs 3.08, 0.82, 1.34, respectively). All types of lymphomas are more common among boys than girls.

Table 1. Population, Under 15 Years of Age, Khon Kaen, Thailand

\begin{tabular}{lrrrrrr}
\hline $\begin{array}{l}\text { Age } \\
\text { group }\end{array}$ & 1990 & 2000 & 2010 & 1990 & 2000 & \multicolumn{1}{c}{2010} \\
\hline 0-4 & 73,394 & 69,218 & 51,900 & 69,746 & 64,886 & 48,000 \\
9-May & 89,058 & 78,230 & 58,000 & 85,308 & 73,311 & 55,300 \\
14-Oct & 96,632 & 76,926 & 65,000 & 90,826 & 72,778 & 62,500 \\
Total & 259,084 & 224,374 & 174,900 & 245,880 & 210,975 & 165,800 \\
\hline
\end{tabular}

Table 2. Number of Cases by Sex and Duration, between 1985-2009 in Khon Kaen, Thailand

\begin{tabular}{lrccc}
\hline \multicolumn{2}{c}{ Year of diagnosis } & \multicolumn{2}{c}{ Males } & \multicolumn{2}{c}{ Females } \\
& Number & $\%$ & Number & $\%$ \\
\hline $1985-1989$ & 96 & 19 & 68 & 18 \\
$1990-1994$ & 102 & 20 & 76 & 20 \\
$1995-1999$ & 110 & 21 & 91 & 24 \\
$2000-2004$ & 114 & 22 & 86 & 22 \\
$2005-2009$ & 92 & 18 & 65 & 17 \\
\hline
\end{tabular}


Table 3. Childhood Cancer Incidences for Both Sexes between 1985-2009 in Khon Kaen, Thailand

\begin{tabular}{|c|c|c|c|c|c|c|}
\hline ICCC group & 1985-1989 & 1990-1994 & 1995-1999 & $2000-2004$ & $2005-2009$ & $1985-2009$ \\
\hline Leukemias, myeloproliferative diseases and others & 28 & 27.8 & 41.1 & 41.9 & 35 & 33.8 \\
\hline Lymphomas and reticuloendothelial neoplasms & 7.2 & 6.5 & 7.8 & 8 & 5.8 & 7 \\
\hline CNS and miscellaneous intracranial and intraspinal neoplasms & 7.3 & 15.8 & 17 & 13 & 12.1 & 12.8 \\
\hline Neuroblastoma and other peripheral nervous cell tumors & 1.4 & 1.6 & 4.5 & 4.8 & 6.4 & 3.8 \\
\hline Retinoblastoma & 3 & 4.2 & 1.1 & 7.6 & 6.9 & 4.6 \\
\hline Renal tumor & 4.1 & 5.3 & 2.1 & 4.1 & 4.1 & 4.1 \\
\hline Hepatic tumors & 1.6 & 3.4 & 1 & 2.3 & 1.4 & 2.1 \\
\hline Malignant bone tumors & 2.4 & 1.6 & 2.5 & 2.3 & 3.1 & 2.6 \\
\hline Soft tissue and other extra-osseous sarcomas & 1.5 & 4.8 & 4.3 & 7 & 4.7 & 4.6 \\
\hline Germ cell tumors, trophoblastic tumors, and others & 2 & 2.2 & 5.1 & 4.3 & 5.3 & 4 \\
\hline Overall incidence (all cancers) & 58.1 & 72.2 & 92.7 & 99.3 & 88.7 & 83 \\
\hline
\end{tabular}

Table 4. Survival Rates of Childhood Cancers between 1985-2009 in Khon Kaen, Thailand

\begin{tabular}{|c|c|c|}
\hline ICCC gr & ival $(\%)$ & $95 \% \mathrm{CI}$ \\
\hline Leukemias, myeloproliferative diseases and others & 49 & $0.43-0.55$ \\
\hline Lymphomas and reticulo-endothelial neoplasms & 63 & $0.52-0.76$ \\
\hline \multicolumn{3}{|c|}{ CNS and miscellaneous intracranial and intra-spinal neoplasms } \\
\hline & 44 & $0.36-0.53$ \\
\hline \multicolumn{3}{|l|}{ Neuroblastoma and other peripheral nervous cell tumors } \\
\hline & 25 & $0.12-0.52$ \\
\hline Retinoblastoma & 55 & $0.40-0.75$ \\
\hline Renal tumor & 67 & $0.51-0.89$ \\
\hline Hepatic tumors & 34 & $0.16-0.72$ \\
\hline Malignant bone tumors & 36 & $0.20-0.62$ \\
\hline Soft tissue and other extra-osseous sarcomas & 46 & $0.32-0.62$ \\
\hline Germ cell tumors, trophoblastic tumors, and others & 74 & $0.62-0.88$ \\
\hline All groups & 52 & $0.48-0.55$ \\
\hline
\end{tabular}

Table 5. Survival Rates of Children with Acute Lymphoblastic and Acute Non-Lymphoblastic Leukemia between 1985-2009 in Khon Kaen, Thailand

\begin{tabular}{lcl}
\hline ICCC group & Survival (\%) & 95\% CI \\
\hline Acute lymphoblastic leukemia & & \\
$1985-2009$ & 51 & $0.45-0.59$ \\
$1985-1995$ & 35 & $0.26-0.49$ \\
$1996-2005$ & 56 & $0.47-0.66$ \\
2006-2009 & 70 & $0.54-0.92$ \\
Acute myeloblastic leukemia & & \\
1985-2009 & 37 & $0.26-0.53$ \\
1985-1995 & 21 & $0.09-0.48$ \\
$1996-2005$ & 45 & $0.28-0.71$ \\
$2006-2009$ & 34 & $0.11-1$ \\
\hline
\end{tabular}

Survival

The probability of 5-year overall survival (OS) was 52\% (95\%CI: $0.48-0.55$ ). Survival differed by type of cancer. The best outcome with highest overall survival was for germ cell tumors (74\%) while the worst outcome was for sympathetic tumors (neuroblastoma) (25\%) (Table 4).

The 5-year overall survival for leukemia was $49 \%$ (1985-2009) (Table 4, Figure 3). The respective survival for ALL and ANLL (1985-2009) was 51\% and 37\%. The survival for ALL had increased from $35 \%$ between 1985-95 to $56 \%$ between $1996-2005$ to $70 \%$ between 2006-9. By comparison, survival rates of ANLL remained unchanged (Table 5).

The overall 5-year survival for lymphoma was $63 \%$ (95\%CI: 0.52 - 0.76). The survival for Hodgkin disease is better than for non-Hodgkin lymphoma (albeit nonsignificantly; $\mathrm{p}=0.45$ ) (Figure 4).

The respective 5-year overall survival for (1) germ

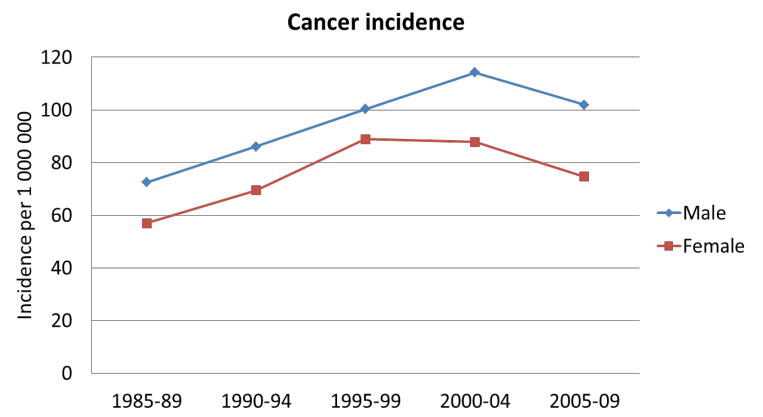

Figure 2. Childhood Cancer Incidences between 19852009 in Khon Kaen, Thailand
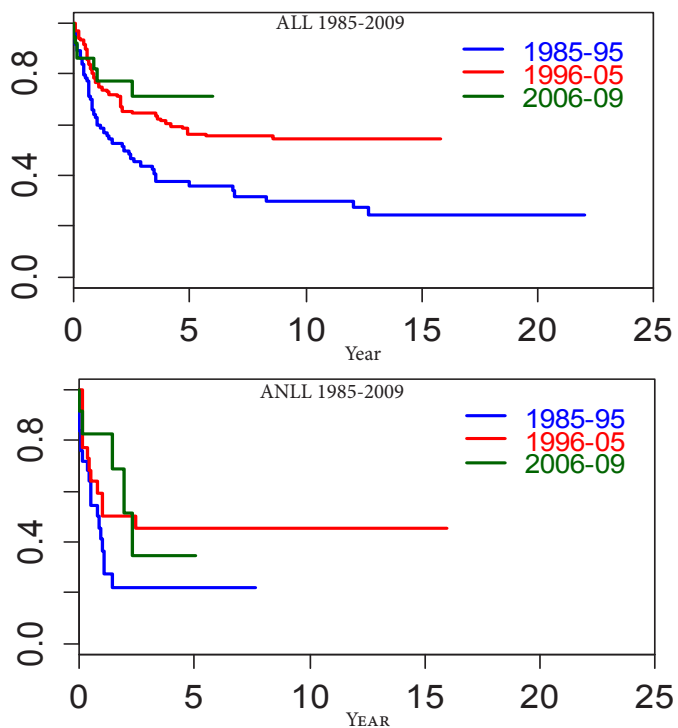

Figure 3. Survival Rates of Children with Acute Lymphoblastic and Myeloblastic Leukemia, by 10-year Interval between 1985-2009 in Khon Kaen, Thailand

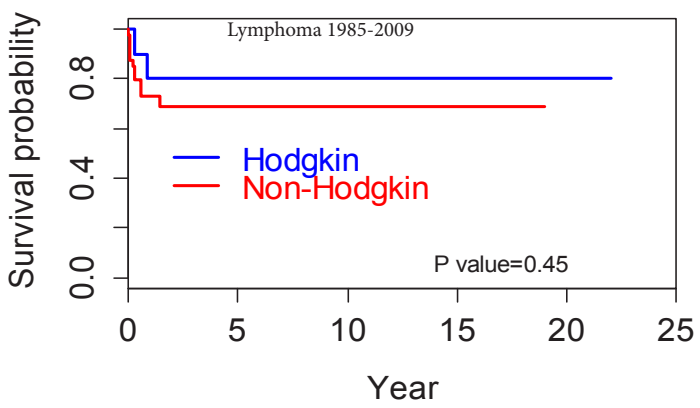

Figure 4. Survival Rates of Children with Hodgkin Disease and Non-Hodgkin Lymphoma between 19852009 in Khon Kaen, Thailand 

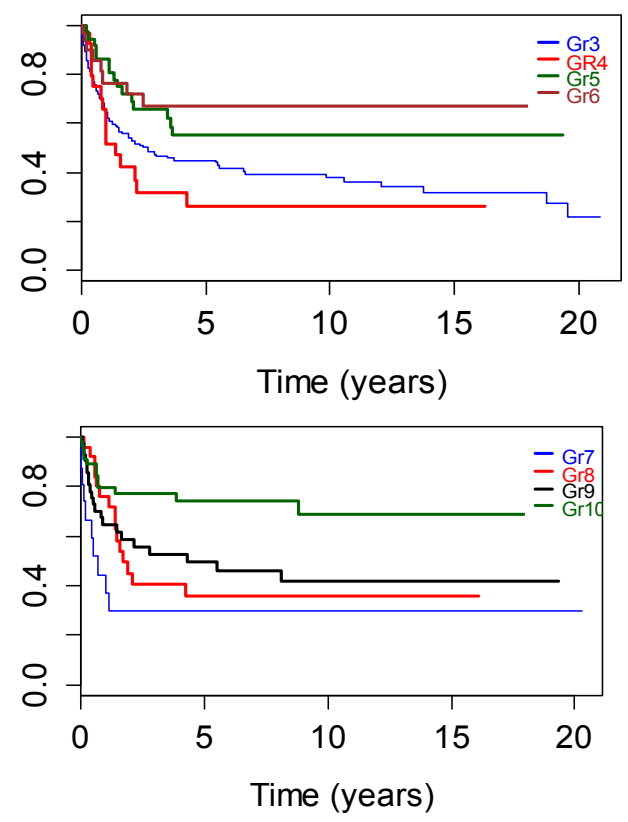

Figure 5. Survival Rates of Children with Solid Tumors between 1985-2009 in Khon Kaen, Thailand. Gr3, CNS tumor; Gr4, neuroblastoma; Gr5, retinoblastoma; Gr6, renal tumors; Gr7, hepatic tumors; Gr8, bone tumors; Gr9, soft tissue sarcoma; Gr10, germ cell tumors

cell tumors, (2) renal tumors, (3) retinoblastoma, (4) soft tissue tumors, (5) CNS tumors, (6) bone tumors, (7) liver tumors, and (8) sympathetic tumor (neuroblastoma) was (1) $74 \%$, , (2) $67 \%$, , (3) $55 \%$, (4) $46 \%$, (5) 44\%, (6) $36 \%$, (7) $34 \%$ and (8) $25 \%$.

\section{Discussion}

This is the first study in Thailand to survey the incidence of all types of childhood cancer over an extended period from a unique population in Khon Kaen province. The microscopic verification (MV) of all cancers was satisfactorily high $(83.7 \%)$ even though the MV for CNS tumors was limited (52.4\%). The age-standardized rate (ASR, 83 per million) was in the range of those extrapolated from the population-based incidences of 4 registries between 1988-1994 (ASR 70.7) (Vattanasapt, 1999) and 1995-1997 (ASR 93) (Wiangnon et al., 2003). The incidence reported by the ThaiPOG for the whole country survey between 2003-5 was 74.9 (Wiangnon et al., 2011). The respective incidence of childhood cancer in Khon Kaen between 1988-1991 and 1985-2000 was 58.7 and 73 per million (Sriamporn et al., 1996; Wiangnon et al., 2004). These incidences were lower than the incidence in this study, which may reflect under-coverage; especially during the early registration era. Notwithstanding the longterm registration, the incidence was still lower than rates reported for western countries albeit comparable to other Southeast Asian countries (Parkin et al., 1998).

As in childhood cancer elsewhere, leukemia is the most common cancer, for which the incidence in Khon Kaen is similar to those of resource-rich countries (Ries et al., 1999). Notably, the incidence of brain tumor was low even though the neuroimaging equipment in Thailand has not been a limiting factor during the last 20 years. Some patients, however, may not have been fully investigated using this modality, resulting in under diagnosis of CNS tumors. Microscopic verification was also rather low for this group of tumors.

Bone tumor incidences were low compared to those of western countries and resource-rich countries in Asia. Generally, bone tumors are more common in adolescents and young adults and we limited the age of children up to 15 . Lymphoma incidence was also low without a clear explanation.

Childhood cancer is becoming an important cause of death in Thailand as poverty-related causes are declining (Sutra et al., 2009). Over the past two decades, childhood cancer mortality has declined dramatically in resource rich countries (Ries et al., 1999; Gatta et al., 2005). The decrease over this period is partially due to the effects of (a) understanding tumor biology and (b) improvements in diagnosis and therapy (Redner, 2011).

Overall survival (OS) of all childhood cancers in the current analysis was lower than those of developed countries; however, it was comparable to population-based survival in Osaka (Ajiki et al., 2004), Thailand (52 vs 54.9) (Wiangnon et al., 2011) and in Khon Kaen at a university hospital (Kamsa-ard et al., 2004). The study of survival of childhood cancers in a population-based in Thailand was unable to identify any trends because of the short duration of the study (i.e., between 2003-5).

Mortality from childhood cancer between 1975-1995 in the USA decreased by $40 \%$ (Ries et al., 1999). Indeed, the outcome has been improving for all types of cancer, especially for leukemia and lymphoma. The five-year survival of lymphoid leukemia has surpassed $80 \%$ in the Nordic countries, Western and Southern Europe, the United Kingdom, and West Germany (Gatta et al., 2005). Our outcomes for leukemia remain low, especially for ANLL. The survival of ALL patients has been gradually increasing from $35 \%$ (1985-95) to $70 \%$ (2006-9). The survival of ANLL patients remains inferior (21-45\%).

Abandonment of chemotherapy was the most common cause of treatment failure, which was strongly related to poor socioeconomic status and financial support. In 2003, the National Security Office (NHSO) implemented universal health coverage nationwide and since then adherence to intensive cancer treatment has significantly risen. In 2006, the NHSO provided and supported a disease management program for the treatment of childhood leukemia and lymphoma. Common treatment protocols were used nationwide. In principle, risk stratification and monitoring of leukemia diagnosis and treatment was obligatory using flow cytometry. In addition, the use of chemotherapy also increased over the period together with overall improvement of supportive care, which resulted in the improvement of ALL survival to $73 \%$ (Seksarn et al., 2011). Notwithstanding, the survival of ANLL was not satisfactorily improved at the national level (Seksarn et al., 2011). Nowadays and importantly, the quality of life is also evaluated for these cancer survivors (Pakakasama et al., 2010).

The two referral-hospitals in Khon Kaen province have pediatric oncologists treating childhood cancers. According to the Thai national referral system, all cases 
residing in Khon Kaen must be referred to one of these 2 centers, in which institutional protocols are initially used. In 2000, many treatment protocols in our institution were revised and developed by modifying the standard international chemotherapy protocol. These were used until the national protocol was implemented in 2006. Survival of ALL was significantly improved during this change.

Among the solid tumors, the highest 5-year OS was observed for germ cell tumor (74\%) followed by Wilms' tumor (67\%), retinoblastoma (55\%), rhabdomyosarcoma (46\%), CNS tumor (44\%), bone tumor (36\%), hepatoblastoma (34\%), and neuroblastoma $(25 \%)$. In general, the OS rates are still not comparable to those reported for resource-rich countries (Ries et al., 1999), except for population-based settings (Ajiki et al., 2004). Survival rates, however, remain below progressive international standards (Gatta et al., 2005).

Recently, the Thai Pediatric Oncology Group proposed common protocols for nationwide use. Improvement of survival and quality of life for children with cancers including survivors are expected (ThaiPOG, 2014).

In conclusion, the incidence and survival rates of childhood cancer in Khon Kaen are lower than those reported for western countries. Survival is, nevertheless, increasing over time after implementation of national protocol and improvement of supportive care facilities. Quality of life of childhood survivors needs to be studied systematically..

\section{Acknowledgements}

The authors thank the staff at the Khon Kaen Cancer Registry for providing the population-based cancer registration data, and Mr. Bryan Roderick Hamman for assistance with English language presentation of the manuscript.

\section{References}

Ajiki W, Tsukuma H, Oshima A (2004). Survival rates of childhood cancer patients in Osaka, Japan. Jpn J Clin Oncol, 34, 50-4.

Gatta G, Capocaccia R, Stiller C, et al (2005). Childhood cancer survival trends in Europe: A EUROCARE Working group study. J Clin Oncol, 23, 3742-51.

Jensen OM, Parkin DM, Maclennan R, Muir CS, Skeet RG, Eds (1991). Cancer registration: Principles and Method. IARC Scientific Publications No.95, Lyon: International Agency for Research on Cancer. 159-76.

Kamsa-ard S, Wiangnon S, Suwanrungruang K, et al (2004). Khon Kaen cancer registry: survival of childhood malignancies recorded between 1993-1997. Thai J Pediatr, 43, 25-31.

Kramarova E, Stiller CA, Ferlay J, Parkin DM, et al (1996). The international classification of childhood cancer. Int $J$ Cancer, 68, 759-65.

National Statistic Office. Statistical data bank and information dissemination division (2003). The 2000 population and housing census. Office of prime minister.

Pakakasama S, Veerakul G, Sosotthikul D, et al (2010). Late effects in survivors of childhood acute lymphoblastic leukemia: a study from Thai Pediatric Oncology Group. Int
J Hematol, 91, 850-4.

Parkin DM, Kramarova E, Draper GJ, et al (1998). International incidence of childhood cancer. Vol II. IARC scientific publication, 144.

Redner A (2011). Leukemias. In: Manual of pediatric hematology and oncology. Lanzkowsky P. 5th ed. Elsevier, Amsterdam. p, 518-66.

Ries LAG, Smith MA, Gurney JG, et al (eds) (1999). Cancer incidence and survival among children and adolescents: United States SEER program 1975-1995, National cancer institute, SEER program. NIH Pub, 99-4649. Bethesda, MD.

Seksarn P (2011). Outcome of childhood leukemia, ThaiPOG study. St. Jude-VIVA forum in pediatric oncology, Singapore.

Sriamporn S, Vattanasapt V, Martin N, et al (1996). Incidence of childhood cancer in Thailand 1988-1991. Paediatr Perinat Epidemiol, 10, 73-85.

Sutra S, Chirawatkul A, Bundhamcharoen K, Ekachampaka P, Wattanamano N (2009). Cause of death analysis by age group. In: Child and adolescent health situation 2009. Working group for child and adolescent health situation in Thailand. The Royal College of Pediatricians of Thailand. Beyond Enterprise, 17-88.

Thai Pediatric Oncology Group (2014). National protocol for treatment of childhood cancer 2014. Bangkok, M Print Corporation.

Vatanasapt V (1999). Childhood cancer. In: Cancer in Thailand 1992-1994. Deerasamee S, Martin N, Sriplung H, et al. eds; IARC No 34, Lyon, 81-6.

Wiangnon S, Kamsa-ard S, Jetsrisuparb A, et al (2003). Childhood cancer in Thailand 1995-1997. Asian Pac J Cancer Prev, 4, 237-43.

Wiangnon S, Kamsa-ard S, Jetsrisuparb A, et al (2004). Childhood malignancies at Khon Kaen, Thailand: population-based cancer registry. Thai J Pediatr, 43, 38-43.

Wiangnon S, Veerakul G, Nuchprayoon I, et al (2011). Childhood cancer incidence and survival 2003-5, Thailand: study from the Thai Pediatric Oncology Group. Asian Pac J Cancer Prev, 12, 2215-20.

World Health Organization (2000). In: International classification of diseases for oncology. Fritz A, Percy C, Jack A, Shanmugaratnam K, Sobin L, Parkin DM, Whelan S, eds. $3^{\text {rd }}$ ed. Geneva. 